\title{
Qualidade do sono e fatores associados em universitários de enfermagem
}

Sleep quality and associated factors in nursing undergraduates Calidad del sueño y factores relacionados en estudiantes universitarios de Enfermería

\author{
Andréia Ferreira dos Santos ${ }^{1}$ (1) https://orcid.org/0000-0003-3567-696x \\ Fernanda Carneiro Mussi' ${ }^{1}$ i https://orcid.org/0000-0003-0692-5912 \\ Claúdia Geovana da Silva Pires ${ }^{1}$ in nttps://orcid.org/0000-0001-9309-2810 \\ Carlos Antônio de Souza Teles Santos ${ }^{1}$ ib https://orcid.org/0000-0003-0970-0479 \\ Melissa Almeida Santos Paim ${ }^{1}$ iD https://orcid.org/0000-0003-3198-3081
}

Como citar:

Santos AF, Mussi FC, Pires CG, Santos CA, Paim MA. Qualidade do sono e fatores associados em universitários de enfermagem. Acta Paul Enferm. 2020;33:eAPE20190144.

DOI

http://dx.doi.org/10.37689/actaape/2020A00144

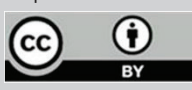

Descritores

Distúrbios do sono-vigeilia; Tabagismo; Estresse psicológico; Fatores de risco; Estudantes; Promoção da saúde

Keywords

Sleep wake disorders; Tabacco user disorder; Stress, psychological; Risk factors; Students; Health promotion

Descriptores

Trastornos del sueño-vigilia; Tabaquismo; Estrés psicológico; Fatores de riesgo; Estudantes: Promoción de la salud

Submetido

11 de Junho de 2019

Aceito

7 de Outubro de 2019

\section{Autor correspondente \\ Fernanda Carneiro Mussi E-mail: femussi@uol.com.br}

\section{Resumo}

Objetivo: Verificar a associação entre tabagismo, estresse, variáveis sociodemográficas e acadêmicas e a qualidade do sono de universitários de enfermagem.

Métodos: Estudo transversal, com 286 universitários de uma instituição pública de ensino superior, em Salvador/ BA. Aplicou-se a Escala de Qualidade do Sono de Pittsburgh, a Escala de Estresse Percebido e instrumentos sobre tabagismo, variáveis sociodemográficas e acadêmicas. Na análise bivariada utilizou-se o teste Qui-quadrado de Pearson ou Exato de Fisher e as variáveis com valor de $p \leq 0,20$ entraram na análise multivariada utilizando-se 0 Modelo de Regressão de Poisson Robusto. Adotou-se significância estatística de 5\%. A modelagem foi realizada com o procedimento backward e para escolha do modelo utilizou-se o critério de informação de Akaike.

Resultados: Na análise múltipla, universitários com renda familiar mensal inferior a quatro salários mínimos tiveram aumento de $20 \%$ na qualidade de sono ruim quando comparados aqueles com renda maior a sete salários (RP: 1,20; IC 95\% 1,01;1,43). Aqueles com alto nível de estresse percebido tiveram aumento de $11 \%$ na qualidade do sono ruim em comparação com aqueles com baixo nível (RP: 1,11; IC 95\% 1,02;1,20). Fumantes/ex-fumantes também tiveram aumento de $11 \%$ na qualidade de sono ruim quando comparados aos não fumantes (RP:1,11; IC 95\% 1,04;1,18). 0 modelo foi ajustado por carga horária no curso e idade.

Conclusão: A baixa renda, o nível alto de estresse e 0 tabagismo foram associados a qualidade do sono ruim. Os resultados desafiam a proposição de intervenções capazes de minimizar a qualidade do sono ruim em universitários de enfermagem.

\section{Abstract}

Objective: To verify the association between smoking, stress, sociodemographic and academic variables and sleep quality of nursing undergraduates.

Methods: Cross-sectional study with 286 undergraduates from a public higher education institution in the city of Salvador, state of Bahia. The Pittsburgh Sleep Quality Scale, the Perceived Stress Scale and tools on smoking, sociodemographic and academic variables were applied. Bivariate analysis was performed using Pearson's chi-square test or Fisher's exact test, and variables with $p$ values $<0.20$ entered the multivariate analysis using the Poisson Robust Regression Model. Statistical significance of $5 \%$ was adopted. The modeling was performed with the backward procedure and the model was chosen using the Akaike information criterion.

Results: In the multiple analysis, undergraduates with a monthly family income of less than four minimum wages had a $20 \%$ increase in poor sleep quality when compared to those with a higher income than seven minimum wages (PR: 1.20; $95 \% \mathrm{Cl} 1.01 ; 1.43$ ). Those with high level of perceived stress had an $11 \%$ increase in poor sleep quality compared with those with low level (PR: 1.11; 95\% Cl 1.02; 1.20). Smokers/ 
former smokers also had an $11 \%$ increase in poor sleep quality compared to non-smokers (PR: $1.11 ; 95 \% \mathrm{Cl} 1.04 ; 1.18$ ). The model was adjusted by course load and age.

Conclusion: Low income, high stress level and smoking were associated with poor sleep quality. The results challenge the proposition of interventions that can minimize the quality of bad sleep in nursing undergraduates.

\section{Resumen}

Objetivo: Verificar la relación entre tabaquismo, estrés y variables sociodemográficas y académicas y la calidad del sueño en estudiantes universitarios de Enfermería. Métodos: Estudio transversal, con 286 estudiantes universitarios de una institución pública de educación superior en Salvador, estado de Bahia. Se aplicó el Índice de Calidad del Sueño de Pittsburgh, la Escala de Estrés Percibido e instrumentos sobre tabaquismo y variables sociodemográficas y académicas. En el análisis bivariado se utilizó la prueba $\chi^{2}$ de Pearson o la prueba exacta de Fisher. Las variables con valor de $p \leq 0,20$ se incluyeron en el análisis multivariado, en el que se utilizó el Modelo de Regresión de Poisson Robusto. Se adoptó significación estadística de 5\%. El modelo se realizó con el procedimiento backward y para elegir el modelo se utilizó el criterio de información de Akaike.

Resultados: En el análisis múltiple, estudiantes universitarios con ingresos familiares inferiores a cuatro salarios mínimos tuvieron un aumento del $20 \%$ en el sueño de mala calidad en comparación con aquellos con ingresos mayores a siete salarios (RP: 1,20; IC 95\% 1,01;1,43). Aquellos con un alto nivel de estrés percibido tuvieron un aumento del $11 \%$ en el sueño de mala calidad en comparación con los de bajo nivel (RP: 1,11; IC 95\% 1,02;1,20). Fumadores/exfumadores también tuvieron un aumento del 11\% en el sueño de mala calidad en comparación con los no fumadores (RP:1,11; IC 95\% 1,04;1,18). El modelo se ajustó por carga horaria de la carrera y edad.

Conclusión: Los ingresos bajos, el alto nivel de estrés y el tabaquismo se relacionaron con el sueño de mala calidad. Los resultados muestran un desafío para proponer intervenciones capaces de minimizar el sueño de mala calidad en estudiantes universitarios de Enfermería.

\section{Introdução}

A quantidade e/ou a qualidade do sono estão envolvidas com a manifestação de diversas alteraçôes cognitivas, psicológicas, imunológicas e metabólicas e são influenciadas por aspectos sociais, clínicos e culturais. ${ }^{(1)}$ A qualidade do sono ruim é um marcador da saúde cardiovascular, sendo o mecanismo inflamatório a resposta fisiológica mais provável. ${ }^{(2,3)}$

Diversos estudos reforçaram as consequências relacionadas ao comprometimento do sono em universitários, como prejuízos no desempenho acadêmico $^{(4,5)}$ e a sonolência excessiva diurna. ${ }^{(6,7)}$

Os universitários são grupos sociais vulneráveis às alteraçóes na qualidade do sono, pois interagem com fatores relacionados à sua privação ao ingressarem no curso. ${ }^{(8)}$ Entre eles, destacam-se o tempo dedicado às atividades acadêmicas; ${ }^{(6,9,10-12)}$ o trabalho em turnos opostos, ${ }^{(10)}$ a ansiedade, a depressão e o estresse, ${ }^{(7,9)} \mathrm{o}$ uso excessivo de redes sociais, telefone celular e televisão ${ }^{(13,14)}$ e o consumo de bebidas alcoólicas. ${ }^{(15)}$

Além das atividades curriculares em horário integral, em busca de qualificação profissional, complementam a formação com atividades extracurriculares, participando de cursos, ligas acadêmicas, estágios, programas de iniciação científica, extensão e monitorias, o que exige maior disponibilidade de tempo para o seu cumprimento. ${ }^{(6)}$ Outros fatores que também estão implicados na qualidade do sono relacionam-se ao sexo, a idade e a situação conjugal. ${ }^{(9,10)}$
Apesar da existência de estudos sobre a qualidade de sono em universitários de enfermagem evidenciando que são maus dormidores, ${ }^{(9,12,16)}$ esses foram realizados em poucos estados brasileiros, com amostras restritas que incluíram apenas os dois semestres iniciais ${ }^{(16)} \mathrm{e}$ ingressantes e concluintes. ${ }^{(12)}$ Pouco se discute sobre a qualidade de sono no ambiente acadêmico e os fatores associados, assim como as medidas de apoio ao discente. ${ }^{(12)}$

Desse modo, é relevante conhecer a qualidade do sono e os principais fatores associados, oferecendo informaçôes sobre aspectos de vulnerabilidade e proteção de universitários. Esse conhecimento pode nortear ações e políticas de promoção à saúde e qualidade de vida durante a formação acadêmica e contribuir para o avanço no campo teórico sobre o tema.

Diante do exposto, o presente estudo objetivou verificar a associação entre tabagismo, estresse, variáveis sociodemográficas e acadêmicas e a qualidade do sono em universitários de enfermagem.

\section{Métodos}

Pesquisa transversal, recorte do projeto matriz "Fatores de risco cardiovascular em graduandos (as) de enfermagem: implicaçóes para o cuidado à saúde", realizado com universitários de enfermagem de uma instituição pública de Salvador, Bahia. Nesta instituição, as atividades curriculares são desenvolvidas nos turnos matutino e vespertino, concentrando-se no matutino. 
O número de dias e turnos do universitário na escola pode variar de acordo com a carga horária semestral. O período de permanência do universitário na escola está também relacionado as atividades extracurriculares e ao número de horas de estudo dedicadas ao curso, além da carga horária semestral.

Os critérios de inclusão definidos para esta investigação foram: universitários de enfermagem, matriculados do primeiro ao décimo semestre do curso, de ambos os sexos e com idade mínima de 18 anos. O critério de exclusão foi afastamento do curso por trancamento ou intercâmbio.

A coleta de dados foi realizada no período de fevereiro de 2016 a março de 2017. Nesse período, 353 universitários estavam matriculados no curso com a seguinte distribuição nos semestres letivos: 48 no primeiro, 39 no segundo, 18 no terceiro, 32 no quarto, 34 no quinto, 34 no sexto, 39 no sétimo, 29 no oitavo, 36 no nono e 44 no décimo. Do total de universitários, 286 aceitarem participar da pesquisa, representando $81,0 \%$ da populaçáo do estudo.

Os universitários foram abordados em sala de aula, no turno matutino, em horário previamente acordado com o docente e o colegiado do curso, onde ocorreu a apresentação dos pesquisadores, a sensibilizaçáo sobre a importância da pesquisa, a explicitação dos objetivos, da operacionalizaçáo da coleta dos dados e do Termo de Consentimento Livre e Esclarecido (TCLE). Os instrumentos foram respondidos em sala de aula após aquiescência a pesquisa e assinatura do TCLE e os pesquisadores aguardaram em sala até o término do preenchimento dos instrumentos.

Utilizou-se um instrumento de caracterizaçấo sociodemográfica com perguntas fechadas e semiestruturadas sobre idade, sexo, raça/cor autodeclarada, situação conjugal, renda familiar mensal e consideraçấo da renda mensal suficiente. Outro instrumento levantou variáveis acadêmicas, como semestre em curso, carga horária cursada no semestre, número de dias e de turnos que frequenta à escola e/ou atividades relacionadas ao curso e número de horas que dispende para atividades acadêmicas além da carga horária semestral. ${ }^{(17)}$ Utilizou-se também questōes referentes ao hábito de fumar (tabagismo atual, ex-fumante e não fumante).

Adotou-se a Escala de Estresse Percebido (PSS), construída por Cohen et al. ${ }^{(18)}$ e traduzida e validada por
Luft et al. ${ }^{(19)}$ no Brasil. Um outro estudo brasileiro realizou a adaptação da escala para universitárias brasileiras, sendo essa versão utilizada nesse estudo. A PSS 10 contém perguntas sobre os sentimentos e pensamentos no último mês, sendo adotada a escala com 10 itens, no formato likert, com cinco níveis de resposta: $0=$ nunca; $1=$ quase nunca; $2=$ às vezes; $3=$ quase sempre; $4=$ sempre). O nível de estresse percebido é classificado em baixo e alto. Valores acima do percentil 75 (40 pontos) são considerados indicativos de alto nível de estresse. ${ }^{(20)}$

Para a avaliação da qualidade do sono, utilizou-se o Índice da Qualidade do Sono de Pittsburgh (PSQI), traduzido, adaptado e validado para a língua portuguesa, ${ }^{(21)}$ o qual mensura a qualidade subjetiva do sono. É válido e confiável( ${ }^{(22)}$ e contém 19 questôes distribuídas em sete domínios: qualidade subjetiva do sono (percepção individual sobre a qualidade do sono, latência do sono (tempo necessário para iniciar o sono), duração do sono (tempo que se permanece dormindo), eficiência habitual do sono (relação entre o número de horas dormidas e de horas de permanência no leito, não necessariamente dormindo), distúrbios do sono (presença de situaçóes que comprometem a hora do sono), uso de medicação para dormir, sonolência diurna e distúrbios durante o dia (refere-se as alteraçôes na disposição e entusiasmo para as atividades rotineiras). Os escores dos domínios são somados para conferir uma pontuaçáo global que varia de 0 a 21 . A qualidade do sono é classificada em boa (pontuaçáo 0-4) ou ruim (pontuação $\geq 5$ ). Quanto maior a pontuação, pior a qualidade do sono. Escores superiores a cinco pontos classifica o indivíduo como mau dormidor, pois indica importante disfunção em pelo menos dois domínios ou disfunção moderada em pelo menos três.

Os dados foram analisados no software estatístico Statistical Package of Social Science versão 20.0. As variáveis categóricas foram analisadas em frequências absolutas e relativas e as contínuas em média e desvio padrăo. Para verificar a associação entre a qualidade do sono e as variáveis de interesse utilizou-se o teste Quiquadrado de Pearson ou Exato de Fisher. Na análise bivariada empregou-se também a razão de prevalência com o respectivo intervalo de confiança (IC) a $95 \%$. As variáveis que obtiveram valor de $\mathrm{p} \leq 0,20$ entraram na análise multivariada utilizando-se o Modelo de Regressão de Poisson Robusto. Foram consideradas 
variáveis de ajustes: idade e carga horária cursada no semestre. $\mathrm{Na}$ análise multivariada, adotou-se significância estatística de 5\%. A modelagem foi realizada com o procedimento backward. Para escolha do modelo foi utilizado o Critério de Informação de Akaike (AIC) escolhendo-se o com menor valor.

A pesquisa foi aprovada pelo Comitê de Ética e Pesquisa, sob parecer $n^{\circ} 353.038$, financiada pelo CNPQ, processo número 309092/2015-9. Atendeu as normas nacionais e internacionais de ética em pesquisa com seres humanos.

\section{Resultados}

A média da idade foi de 23,48 anos (DP = 4,421), valor mínimo de 18 e máximo de 50 . Predominaram sexo feminino $(90,2 \%)$, solteiros com companheiros $(90,9 \%)$ e autodeclarados negros $(87,8 \%)$. A maioria não exercia atividade remunerada $(81,5 \%)$, tinha renda familiar mensal menor que quatro salários mínimos $(47,2 \%)$, considerava a renda insatisfatória $(65,0 \%)$ e residia com duas ou três pessoas $(55,6 \%)$.
A maioria cursava entre o $6^{\circ}$ e $10^{\circ}$ semestre $(54,5 \%)$, cumpria carga horária semestral entre 401 a 500 horas $(59,1 \%)$, frequentava a escola mais de quatro dias por semana $(87,1 \%)$ e de dois a três turnos $(80,8 \%)$, dispendia até três horas diárias para realizar outras atividades além da carga horária semestral (58,0\%). Predominaram baixo nível de estresse percebido $(74,5 \%)$ e não fumantes/ex-fumantes $(95,8 \%)$.

A maioria dos universitários tinha qualidade do sono ruim $(86,4 \%)$, a qual foi prevalente para aqueles do sexo feminino $(87,9 \%)$, na faixa etária entre 18 e 21 anos (87,4\%), autodeclarados negros $(86,9 \%)$, com renda familiar mensal menor que quatro salários mínimos $(90,4 \%)$, que consideravam a renda insatisfatória $(88,7 \%)$, separados/ divorciados e viúvos (100,0\%), que residiam sozinhos ou com uma pessoa $(88,3 \%)$ e não trabalhavam $(87,1 \%)$. Na análise bivariada, verificou-se qualidade do sono ruim para universitários do sexo feminino $(p=0,015)$, com renda mensal menor que quatro salários mínimos ( $\mathrm{p}=0,015)$ e que não consideravam a renda satisfatória para a subsistência ( $\mathrm{p}$ $=0,115)$ (Tabela 1).

Tabela 1. Prevalência e Razão de Prevalência (RP) da qualidade do sono ruim em universitários de enfermagem de acordo com características sociodemográficas

\begin{tabular}{|c|c|c|c|c|c|}
\hline Características Sociodemográficas & Total n (\%) & Prevalência (\%) & $\mathrm{p}$-value & $\mathrm{RP}$ & IC 95\% \\
\hline Sexo & & & $0,015^{*}$ & & \\
\hline Masculino & $28(9,8)$ & 71,4 & & & \\
\hline Feminino & $258(90,2)$ & 87,9 & & 1,23 & $(0,96 ; 1,56)$ \\
\hline Faixa etária & & & $0,955^{\star \star}$ & & \\
\hline 18 a 21 anos & $87(30,4)$ & 87,4 & & & \\
\hline 22 a 30 & $185(64,7)$ & 85,9 & & 0,98 & $(0,89 ; 1,08)$ \\
\hline 31 a 50 anos & $14(4,9)$ & 85,7 & & 0,98 & $(0,78 ; 1,23)$ \\
\hline Raça/Cor autodeclarada & & & $0,519^{*}$ & & \\
\hline Branca & $35(12,2)$ & 82,9 & & & \\
\hline Negra & $251(87,8)$ & 86,9 & & 1,04 & $(0,89 ; 1,22)$ \\
\hline 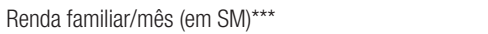 & & & $0,015^{\star}$ & & \\
\hline$>7$ & $50(17,5)$ & 74,0 & & & \\
\hline 4 a 7 & $101(35,3)$ & 87,1 & & 1,17 & $(0,98 ; 1,41)$ \\
\hline$<4$ & $135(47,2)$ & 90,4 & & 1,22 & $(1,02 ; 1,45)$ \\
\hline Satisfação com a renda mensal & & & $0,115^{\star}$ & & \\
\hline Sim & $100(35,0)$ & 82,0 & & & \\
\hline Não & $186(65,0)$ & 88,7 & & 1,08 & $(0,97 ; 1,20)$ \\
\hline Situação conjugal & & & $0,513^{\star \star}$ & & \\
\hline Separado/Divorciado/Niúvo/ Solteiro sem companheiro & $2(0,7)$ & 100,0 & & & \\
\hline Solteiro com companheiro & $260(90,9)$ & 86,9 & & 0,86 & $(0,82 ; 0,91)$ \\
\hline Casado/união estável & $24(8,4)$ & 79,2 & & 0,79 & $(0,64 ; 0,97)$ \\
\hline$N^{\circ}$ pessoas com quem reside & & & $0,500^{*}$ & & \\
\hline Nenhuma/uma & $60(21,0)$ & 88,3 & & & \\
\hline 2 ou 3 & $159(55,6)$ & 87,4 & & 0,98 & $(0,88 ; 1,10)$ \\
\hline$\geq 4$ & $6723,4)$ & 82,1 & & 0,92 & $(0,80 ; 1,07)$ \\
\hline Atividade Remunerada & & & $0,432^{\star}$ & & \\
\hline Não & $233(81,5)$ & 87,1 & & & \\
\hline Sim & $53(18,5)$ & 83,0 & & 0,95 & $(0,83 ; 1,08)$ \\
\hline
\end{tabular}

p-value obtido pelo Qui-quadrado de Pearson* ou Exato de Fischer*; " "'Salário mínimo (SM) da época da pesquisa R\$880,00 (ano 2016) e R\$937,00 (ano 2017); RP - Razão de Prevalência; IC - Intervalo de Confiança 
Tabela 2. Prevalência e Razão de Prevalência (RP) da qualidade do sono ruim em universitários de enfermagem de acordo com variáveis acadêmicas, nível de estresse e tabagismo

\begin{tabular}{|c|c|c|c|c|c|}
\hline Variáveis & Total n (\%) & Prevalência (\%) & $p$-value & $\mathrm{RP}$ & IC $95 \%$ \\
\hline \multicolumn{6}{|l|}{ Acadêmicas } \\
\hline \multicolumn{6}{|l|}{ Semestre em curso } \\
\hline $1^{\circ}$ ao $5^{\circ}$ & $130(45,5)$ & 85,4 & $0,660^{*}$ & & \\
\hline $6^{\circ}$ ao $10^{\circ}$ & $156(54,5)$ & 87,2 & & 1,02 & $(0,93 ; 1,12$ \\
\hline Carga horária nesse semestre & & & $0,296^{*}$ & & \\
\hline 136 a 400 horas & $68(23,8)$ & 80,9 & & & \\
\hline 401 a 500 horas & $169(59,1)$ & 87,6 & & 1,08 & $(0,95 ; 1,23$ \\
\hline$\geq 501$ horas & $49(17,1)$ & 89,8 & & 1,11 & $(0,95 ; 1,28$ \\
\hline$N^{0}$ de dias na universidade por semana & & & $0,981^{*}$ & & \\
\hline 1 a 3 & $37(12,9)$ & 86,5 & & & \\
\hline 4 a 6 & $249(87,1)$ & 86,4 & & 0,99 & $(0,87 ; 1,14$ \\
\hline$N^{N}$ de turnos que frequenta o curso & & & $0,274^{*}$ & & \\
\hline 1 & $55(19,2)$ & 81,8 & & 1,06 & $(0,93 ; 1,22$ \\
\hline 2 a 3 & $231(80,8)$ & 87,5 & & & \\
\hline $\begin{array}{l}\text { Tempo dispendido em atividades além da carga } \\
\text { horária semestral }\end{array}$ & & & $0,128^{*}$ & & \\
\hline Até $3 \mathrm{~h} / \mathrm{dia}$ & $166(58,0)$ & 83,7 & & & \\
\hline$>3 \mathrm{~h} / \mathrm{dia}$ & $120(42,0)$ & 90,0 & & 1,07 & $(0,98 ; 1,17$ \\
\hline Nível de estresse percebido & & & $0,018^{\star *}$ & & \\
\hline Baixo & $213(74,5)$ & 83,6 & & & \\
\hline Alto & $73(25,5)$ & 94,5 & & 1,13 & $(1,04 ; 1,22$ \\
\hline Tabagismo ativo & & & $0,160^{*}$ & & \\
\hline Não / parou & $274(95,8)$ & 85,7 & & & \\
\hline Sim & $12(4,2)$ & 100,00 & & 1,16 & $(1,11 ; 1,22$ \\
\hline
\end{tabular}

p-value obtido pelo Qui-quadrado de Pearson* ou Exato de Fischer*; RP - Razão de Prevalência; IC - Intervalo de Confiança

A qualidade do sono ruim foi também predominantemente constatada para universitários entre o $6^{\circ}$ e o $10^{\circ}$ semestre $(87,2 \%)$, com carga horária maior ou igual a 501 horas no semestre em curso $(89,8 \%)$, que frequentavam a escola de um a três dias por semana $(86,5 \%)$ e em dois ou três turnos $(87,5 \%)$, que dispendiam mais de três horas para realizar atividades do curso além dos turnos que frequentavam a escola $(90,0 \%)$, que estavam com alto nível de estresse $(94,5 \%)$ e eram fumantes ativos $(100,0 \%)$. Na análise bivariada, verificou-se qualidade do sono ruim para universitários que dispendiam mais de três horas em atividades de estudo além da carga horária semestral $(\mathrm{p}=0,128)$, apresentavam nível de estresse alto $(0,018)$ e fumavam ou eram ex-fumantes $(p=0,160)$. A razão de prevalência seguiu a mesma direção (Tabela 2).

$\mathrm{Na}$ análise múltipla, as variáveis que mais contribuíram para a qualidade do sono ruim foram a renda familiar mensal inferior a quatro salários mínimos, o nível de estresse percebido alto e o tabagismo. Universitários com renda familiar mensal inferior a quatro salários mínimos tiveram aumento de $20 \%$ na qualidade de sono ruim quando comparados aqueles com renda maior a sete salários (RP: 1,20; IC 95\% $1,01 ; 1,43)$. Aqueles com alto nível de estresse perce- bido tiveram aumento de $11 \%$ na qualidade do sono ruim em comparação com aqueles com baixo nível (RP: 1,11; IC 95\% 1,02;1,20). Fumantes/ex-fumantes também tiveram aumento de $11 \%$ na qualidade de sono ruim quando comparados aos não fumantes (RP:1,11; IC 95\% 1,04;1,18). O modelo foi ajustado por carga horária no curso e idade (Tabela 3).

Tabela 3. Associação entre as variáveis preditoras da qualidade do sono ruim em universitários de enfermagem

\begin{tabular}{|c|c|c|}
\hline Variáveis & $\mathrm{RP}$ & IC $95 \%$ \\
\hline Idade & & 1,0 \\
\hline \multicolumn{3}{|l|}{18 a 21 anos } \\
\hline 22 a 30 & 1,00 & $(0,90 ; 1,11)$ \\
\hline 31 a 50 anos & 1,04 & $(0,84 ; 1,30)$ \\
\hline Renda familiar/mês (em SM) & & 1,0 \\
\hline \multicolumn{3}{|l|}{$>7$} \\
\hline 4 a 7 & 1,15 & $(0,96 ; 1,37)$ \\
\hline$<4$ & 1,20 & $(1,01 ; 1,43)$ \\
\hline Carga horária nesse semestre & & 1,0 \\
\hline \multicolumn{3}{|l|}{ D136 a 400 horas } \\
\hline 401 a 500 horas & 1,08 & $(0,95 ; 1,23)$ \\
\hline$\geq 501$ horas & 1,10 & $(0,94 ; 1,27)$ \\
\hline Nível de estresse percebido & & 1,0 \\
\hline \multicolumn{3}{|l|}{ Baixo } \\
\hline Alto & 1,11 & $(1,02 ; 1,20)$ \\
\hline Tabagismo ativo & & 1,0 \\
\hline \multicolumn{3}{|l|}{ Não / parou } \\
\hline \multirow[t]{2}{*}{ Sim } & 1,11 & $(1,04 ; 1,18)$ \\
\hline & $A I C=582,18$ & \\
\hline
\end{tabular}

Modelo de Regressão Logística ajustado por idade e carga horária cursada; RP - Razão de Prevalência; IC - Intervalo de Confiança. *Salário mínimo (SM) na época da época da pesquisa $R \$ 880,00$ (ano 2016) e $\mathrm{R} \$ 937,00$ (ano 2017) 


\section{Discussão}

Alteraçóes do sono têm sido associadas, cada vez mais, ao aumento da morbidade e mortalidade. Melhorar a qualidade do sono diminui a incidência de transtornos depressivos, psicoses e doenças cardiovasculares, metabólicas e inflamatórias. ${ }^{(23)} \mathrm{Os}$ riscos à saúde, relacionados a qualidade do sono ruim, torna preocupante a prevalência elevada para os universitários de enfermagem. Corroboram com esse achado outras pesquisas com universitários da área de enfermagem, ${ }^{(9,12,16)}$ de outras profissóes da área da saúde, ${ }^{(4-7,10,13,14,16,24-26)}$ e de outras áreas de formação acadêmica. ${ }^{(11,15)}$

Dado relevante desse estudo mostrou que o alto nível de estresse percebido foi associado a qualidade de sono ruim, corroborando com outras investigaçôes com universitários. ${ }^{(9,26,27)}$ Uma hipótese aventada para essa relação é a de que indivíduos com sintomas de estresse, apresentam níveis séricos elevados do hormônio cortisol, que os mantém ativos e em estado de alerta. Modificaçóes na secreção de cortisol podem estar relacionadas com as queixas típicas associadas aos problemas do sono. ${ }^{(14)}$

Destacam-se como potenciais estressores para os universitários o excesso de responsabilidades acadêmicas, a quantidade elevada de componentes curriculares e carga horária, o envolvimento com atividades extraclasse, as dificuldades em relação aos conteúdos apreendidos, a conciliação entre exigências acadêmicas e o convívio familiar e social, as preocupaçóes com as avaliaçóes e a aquisição de conhecimentos, a incerteza da inserçáo no mercado de trabalho, o deslocamento para o campus universitário, os conflitos interpessoais com os pais, professores e a turma. ${ }^{(9)}$ Nessa investigação, os universitários que dispendiam mais de três horas em atividades, além dos turnos que frequentavam o curso, apresentaram qualidade de sono ruim. Muitas vezes, devido as exigências acadêmicas, utilizam parte do período de sono para atendê-las e, assim sendo, dormem mais tarde e acordam mais cedo, tendo menor tempo de sono durante a noite, geralmente inferior ao necessário para o restabelecimento orgânico. ${ }^{(9)}$

Chamou atenção que universitários entre o sexto e o décimo semestre do curso, tiveram maior pre- valência de qualidade do sono ruim em relação aos que cursavam do primeiro ao quinto semestre, embora a fase de formação não tenha sido uma variável com significância estatística na análise multivariada. Esse achado pode refletir a fase de formação que os colocam mais expostos a variabilidade dos turnos em instituiçóes hospitalares, como ocorre com os universitários em estágio curricular que atuam em atividades práticas em esquema de rodízio de turnos, inclusive noturno. Ademais, nesta fase da formação, mais inseridos na prática clínica, podem estar mais expostos a fatores estressores, como as dificuldades e conflitos na comunicação entre a equipe multiprofissional, a necessidade de desenvolver procedimentos junto aos pacientes e a vivência de sentimentos de sofrimento que surgem no envolvimento com o cuidado. Essas consideraçóes, entre outros aspectos, demandam novas pesquisas para melhor entendimento da relação da fase de formação com as alteraçóes na qualidade do sono.

Evidenciou-se também que, universitários com renda familiar mensal inferior a quatro salários mínimos e que não consideravam a renda satisfatória para a subsistência, apresentaram qualidade do sono ruim. De forma geral, há uma relação entre sono e nível socioeconômico. O baixo nível socioeconômico pode ser um agente estressor e redutor da qualidade do sono. ${ }^{(11)}$ Uma investigação justificou que indivíduos com menor renda, estão expostos a fatores que influenciam negativamente o sono, como as preocupaçóes e o domicílio localizado em regióes barulhentas. ${ }^{(28)}$ Revisão sistemática da literatura realizada com adolescentes de diversos estratos socioeconômicos, constatou relação significante entre os indicadores socioeconômicos e a qualidade do sono, observando que a baixa condiçáo socioeconômica refletiu na pior percepção subjetiva da qualidade de sono, na menor duração do sono e na maior sonolência diurna. ${ }^{(29)}$ No entanto, essa associação ainda é pouco explorada, não sendo encontrado na literatura outras pesquisas envolvendo universitários.

Nesse estudo, as universitárias apresentaram pior qualidade do sono. As mulheres normalmente apresentam alteraçóes do sono relacionadas à influência das variaçôes hormonais fisiológicas e cíclicas, ${ }^{(22)}$ o que também pode levar a maior sus- 
ceptibilidade a fatores externos, como o estresse. ${ }^{(30)}$ Embora as pesquisas demonstrem que as mulheres apresentam sono alterado, ${ }^{(31,32)}$ inclusive em estudos com universitários, ${ }^{(29)}$ ainda não está totalmente esclarecido os mecanismos estruturais e biológicos que explicariam esse fato.

O tabagismo foi também associado a má qualidade do sono. Sabe-se que a nicotina aumenta os níveis séricos da dopamina, neurotransmissor que age como estimulante no organismo, aumentando o estado de alerta e influenciando na manifestação de distúrbios do sono, como a insônia e a sonolência diurna. ${ }^{(33)}$ Outras investigações com universitários também identificaram associação entre qualidade do sono ruim e tabagismo. ${ }^{(24,27)}$

$\mathrm{Na}$ análise multivariada, a renda familiar mensal inferior a quatro salários mínimos, o nível de estresse percebido alto e o tabagismo foram as variáveis que mais contribuíram para a qualidade do sono ruim, evidenciando múltiplos fatores relacionados ao prejuízo da quantidade e/ou qualidade do sono. ${ }^{(34)}$ Esses achados evidenciam que o sono, além de ser regulado por mecanismos fisiológicos, ${ }^{(35)}$ sofre influência de fatores externos, os quais afetaram o grupo estudado.

Face a constatação do grupo de variáveis associadas a qualidade do sono ruim, depreende-se que programas educativos e de apoio psicopedagógico junto aos universitários, podem contribuir para tomarem consciência dos prejuízos à saúde decorrentes da qualidade do sono ruim, para a valorização da preservação da qualidade do sono, melhor gerenciamento do tempo destinado as atividades acadêmicas, melhor enfrentamento dos fatores estressores e conflitos durante a formação acadêmica e, para o controle do tabagismo ativo e passivo. Torna-se fundamental, também, a reflexão dos resultados desse estudo com docentes e gestores educacionais para que possam analisar o impacto das atividades e responsabilidades acadêmicas na qualidade do sono dos universitários, e orientar o planejamento integrado dos componentes curriculares, chegando-se a um equilíbrio entre as metas e as demandas pretendidas da formação.

Destaca-se como limitaçóes da pesquisa, a amostragem por acessibilidade e o estudo transversal, que não permite inferir causalidade, uma vez que exposição e desfecho são coletados simultaneamente. Recomenda-se estudos longitudinais para acompanhar a qualidade do sono de universitários durante as diferentes fases da formação acadêmica.

\section{Conclusão}

Prevaleceram universitários com qualidade do sono ruim. Na análise múltipla, ajustada por idade e carga horária, variáveis psicossociais e comportamentais contribuíram para a qualidade do sono ruim, como a baixa renda familiar mensal, o nível alto de estresse percebido e o tabagismo. Os resultados desafiam a proposição de intervenções capazes de minimizar a qualidade do sono ruim em universitários de enfermagem.

\section{Agradecimentos}

Ao Conselho Nacional de Desenvolvimento de Pesquisa e Tecnologia - CNPQ, pelo financiamento do projeto de Pesquisa "Fatores de risco cardiovascular em graduandos (as) de enfermagem: implicaçôes para o cuidado à saúde”, ao qual o artigo está vinculado.

\section{Colaborações}

Santos AF, Mussi FC, Pires CGS, Santos CAST e Paim MAS contribuíram com a concepção do projeto, revisão de literatura, análise e interpretação dos dados, redação do artigo, revisão crítica relevante do conteúdo intelectual do artigo, adequação às normas da revista e aprovação da versão final a ser publicada.

\section{Referências}

1. Li D, Liu D, Wang X, He D. Self-reported habitual snoring and risk of cardiovascular disease and all-cause mortality. Atherosclerosis. 2014;235(1):189-95.

2. Laks J, Telles L, Leonardo L. Telles, Insônia e doença cardiovascular: marcadores inflamatórios e risco aumentado de cardiopatias. J Bras Med (São Paulo). 2014;02(2):15-9. 
3. Azad MC, Fraser K, Rumana N, Abdullah AF, Shahana N, Hanly PJ, et al. Sleep disturbances among medical students: a global perspective. J Clin Sleep Med. 2015;11(1):69-74.

4. Arbabisarjou A, Hashemi SM, Sharif MR, Haji Alizadeh K, Yarmohammadzadeh P, Feyzollahi Z. The Relationship between Sleep Quality and Social Intimacy, and Academic Burn-Out in Students of Medical Sciences. Glob J Health Sci. 2015;8(5):231-8.

5. Mirghani HO, Mohammed OS, Almurtadha YM, Ahmed MS. Good sleep quality is associated with better academic performance among Sudanese medical students. BMC Res Notes. 2015;8(1):706.

6. Carvalho TMCS, Silva Junior II, Siqueira PPS, Almeida JO, Soares AF, Lima AMJ. Qualidade do Sono e Sonolência Diurna Entre Estudantes Universitários de Diferentes Áreas. Rev Neurociênc. 2013;21(3):383-7.

7. Choueiry N, Salamoun T, Jabbour H, El Osta N, Hajj A, Rabbaa Khabbaz L. Insomnia and relationship with anxiety in university students: a cross-sectional designed study. PLoS One. 2016;11(2):e0149643.

8. Banks S, Dinges DF. Behavioral and physiological consequences of sleep restriction. J Clin Sleep Med. 2007;3(5):519-28.

9. Benavente SBT, Silva RM, Higashi AB, Guido LA, Costa AL. Influência de fatores de estresse e características sociodemográficas na qualidade do sono de estudantes de enfermagem. Rev Esc Enferm USP. 2014;48(3):514-20.

10. Obrecht A, Collaço IAL, Valderramas SR, Miranda KC, Vargas EC, Szkudlarek AC. Análise da qualidade do sono em estudantes de graduação de diferentes turnos. Revi Neurociênc. 2015;23(2):205-10.

11. Felden EP, Ferrari GJ Junior, Andrade RD, Claumann GS, Pelegrini A, Teixeira CS. Fatores associados à baixa duração do sono em universitários ingressantes. Rev Bras Ci Mov. 2015;23(4):94-103.

12. Ferreira SC, Jesus TB, Santos AS. Qualidade do sono e fatores de risco cardiovasculares em acadêmicos de enfermagem. Rev Eletrôn Gestão Saúde. 2015;6(1):390-04.

13. Mohammadbeigi A, Absari R, Valizadeh F, Saadati M, Sharifimoghadam S, Ahmadi A, et al. Sleep quality in medical students; the impact of over-use of mobile cell-phone and social networks. J Res Health Sci. 2016;16(1):46-50.

14. Araújo MF, Vasconcelos HC, Marinho NB, Freitas RW, Damasceno MM. Níveis plasmáticos de Cortisol em universitários com má qualidade de sono. Cad Saúde Colet. 2016;24(1):105-110.

15. Kenney SR, Paves AP, Grimaldi EM, LaBrie JW. Sleep quality and alcohol risk in college students: examining the moderating effects of drinking motives. J Am Coll Health. 2014;62(5):301-8.

16. Araújo MA, Pereira Fabi FW, Bortolonii R, Filho WD, Ilha S, Alvarenga MR. Padrão do sono em estudantes de enfermagem de Universidade Pública Federal. Rev Cubana Enferm. 2016;32(2):162-70.

17. Pires CG, Azevedo SR, Mussi FC. Fatores de risco cardiovascular em est de enfermagem: elaboração de procedimentos de avaliação. Rev Baiana Enferm. 2014;28(3):294-302.

18. Cohen S, Kamarck T, Mermelstein R. A global measure of perceived stress. J Health Soc Behav. 1983;24(4):385-96.

19. Luft CD, Sanches SO, Mazo GZ, Andrade A. Versão brasileira da Escala de Estresse Percebido: tradução e validação para idosos. Rev Saude Publica. 2007;41(4):606-15.
20. Dias JC, Silva WR, Maroco JB, Bonini JA. Escala de Estresse Percebido Aplicada a Estudantes Universitárias: estudo de Validação. Psychol Community Health. 2015;4(1):1-13.

21. Bertolazi AN, Fagondes SC, Hoff LS, Dartora EG, Miozzo IC, de Barba ME, et al. Validation of the Brazilian Portuguese version of the Pittsburgh Sleep Quality Index. Sleep Med. 2011;12(1):70-5.

22. Buysse DJ, Reynolds CF 3rd, Monk TH, Berman SR, Kupfer DJ. The Pittsburgh Sleep Quality Index: a new instrument for psychiatric practice and research. Psychiatry Res. 1989;28(2):193-213.

23. Carroll JE, Seeman TE, Olmstead R, Melendez G, Sadakane R, Bootzin $\mathrm{R}$, et al. Improved sleep quality in older adults with insomnia reduces biomarkers of disease risk: pilot results from a randomized controlled comparative efficacy trial. Psychoneuroendocrinology. 2015;55:18492.

24. Araújo MFM, Freitas RWJF, Lima ACS, Pereira DCR, Zanetti ML, Damasceno MMC. Indicadores de saúde associados com a má qualidade do sono de universitários. Rev Esc Enferm USP. 2014;48(6):1085-92.

25. Ferreira CM, Kluthcovsky AC, Dornelles CF, Stumpf MA, Cordeiro TM. Qualidade do sono em estudantes de medicina de uma universidade do Sul do Brasil. Conexão Ci (Formiga). 2017;12(1):78-85.

26. Costa FF, Freitas AG, Jesus LG, Magalhães FM, Pereira C, Silva GM, et al. Qualidade de sono e estresse em universitários dos últimos semestres dos cursos da área da saúde. E-RAC. 2015;5(2):1-15.

27. Średniawa PA, Drwiła D, Krotos A, Wojtaś D, Kostecka N, Tomasik T. Insomnia and the level of stress among students in Krakow. Trends Psychiatry Psychother. 2019. pii: S2237-60892019005001102. doi: 10.1590/2237-6089-2017-0154.

28. Jarrin DC, McGrath JJ, Quon EC. Objective and subjective socioeconomic gradients exist for sleep in children and adolescents. Health Psychol. 2014;33(3):301-5.

29. Felden EP, Leite CR, Rebelatto CF, Andrade RD, Beltrame TS. Sono em adolescentes de diferentes níveis socioeconômicos: revisão sistemática. Rev Paul Pediatr. 2015;33(4):467-73.

30. Calais SL, Andrade LM, Lipp ME. Diferenças de sexo e escolaridade na manifestação de stress em adultos jovens. Psicol Reflex Crit. 2003;16(2):257-63.

31. Zanuto EA, Lima MC, Araújo RG, Silva EP, Anzolin CC, Araújo MY, et al. Distúrbios do sono em adultos de uma cidade do Estado de São Paulo. Rev Bras Epidemiol. 2015;18(1):42-53.

32. da Silva Timossi L, Leite N, Vecchi Osiecki AC, Fuzetti Cavazza J, Cieslak F, Osiecki R. Tabagismo, consumo alcoólico e tempo de sono em trabalhadores ativos da indústria do estado do Paraná-Brasil. Rev Salud Publica (Bogota). 2014;16(4):491-504.

33. Balbani AP, Montovani JC. Métodos para abandono do tabagismo e tratamento da dependência da nicotina. Rev Bras Otorrinolaringol. 2005;71(6):820-7.

34. Gomes MM, Quinhones MS, Engelhardt E. Neurofisiologia do sono e aspectos farmacoterapêuticos dos seus transtornos. Rev Bras Neurol. 2010;46(1):5-15.

35. Alóe F, Azevedo AP, Hasan R. Mecanismos do ciclo sono-vigília. Braz J Psychiatry. 2005;27(1 Suppl 1):33-9. 